Each patient's electronic and paper notes were reviewed to identify choice and order of intravenous bronchodilator alongside need for ventilatory support as a marker of severity.

These results were reviewed in the context of a local guideline suggesting salbutamol as the first line, magnesium sulphate as the second line and aminophylline as third line.

Results 37 cases of children requiring intravenous bronchodilator were identified. Of these $12(32 \%)$ of children received a single intravenous bronchodilator, 15 (40\%) received two bronchodilators and $10 \quad(27 \%)$ received three different bronchodilators.

In contrast to the guideline, magnesium sulphate was the first choice in 35 (95\%) of cases, with salbutamol chosen first in only $2(5 \%)$ of cases. Aminophylline was only ever used after both magnesium and salbutamol had been commenced.

Of this group $5(13.5 \%)$ received ventilatory support beyond simple oxygen delivery with 3 receiving Optiflow, 1 CPAP, 1 BiPAP and none requiring intubation.

Discussion Variation and uncertainty at national level is reflected in local practice. Use of magnesium sulphate is far higher than suggested by our local guidelines and appears to be driven by individual clinicians' experiences.

This study identifies the need for further work to develop an evidence based approach to managing the child with life threatening wheeze or asthma to limit variation and, it is hoped, improve outcomes.

\section{G89(P) ACCIDENTAL INGESTION OF POTENTIALLY HARMFUL SUBSTANCES: ANALYSING PAEDIATRIC EMERGENCY DEPARTMENT PRESENTATIONS TO GUIDE HEALTH PROMOTION ADVICE AND TRIAGE}

KM Knight, V Merrick. Paediatric Emergency Department, Royal London Hospital, London, UK

\subsection{6/archdischild-2015-308599.88}

Aims Accidental ingestion of a potentially harmful substance is a common complaint in paediatric A\&E - in 2002, 31,500 children $<15$ years old attended hospital with suspected poisoning. Large UK based studies focus on fatal poisonings. However, most children do not require any treatment, and the literature currently lacks detail regarding the minor end of the spectrum.

Gathering data from a large inner city paediatric A\&E, we studied every attendance with accidental ingestion - not only cases requiring intervention - to look for emerging trends to guide health promotion advice, and improve the pathway for these children from triage through to discharge.

Method Two years of electronic records were analysed. Search criteria included any presenting complaint or discharge diagnosis containing the term 'accidental ingestion' or 'accidental overdose'. Demographic data was established, along with details of the substance ingested, any treatment or admission required and any follow up arranged.

Results There were 169 presentations during 2012-2013. 1223 month old children accounted for $29 \%$ of cases. There was no seasonal variation, and the gender divide was equal. In $90 \%$ of cases, the child was unsupervised when the ingestion occurred. Substances were divided by category - parent/carers medication (33\% of cases); bathroom/kitchen product $(21 \%)$; paracetamol containing products $(11 \%)$; rodent/insect poison (8\%), and own medication (5\%). Most children (57\%) were sent home with reassurance. $21 \%$ had 4 h of A\&E observation, while only $8 \%$ were admitted. $3 \%$ returned for repeat tests. Sixteen cases $(9 \%)$ had a documented health visitor referral. Social services were notified about three cases.

Conclusion Although most events happened while the child was unsupervised, there were few health visitor referrals - a key strategy in preventing further incidents. Most substances ingested were medications or cleaning products. Children can sometimes still open 'child resistant' containers, so health promotion advice should focus on safer storage. All children waited to see a doctor, however the majority needed no treatment or observation. There may be scope for certain children to be discharged from nurse triage, streamlining their A\&E pathway.

\section{G90(P) N N 0 LAUGHING MATTER}

P Seddon, K Noble. General Paediatrics, Brighton and Sussex NHS Trust, Brighton, UK

\subsection{6/archdischild-2015-308599.89}

Aims To investigate the risks posed to young people from the recreational use of nitrous oxide ("laughing gas").

Methods We highlight the case of a girl aged 15 who presented to a Children's Emergency Department with significant subcutaneous emphysema, pneumothorax and pneumomediastinum, following nitrous oxide inhalation at a festival the previous day. We reviewed the current literature in order to understand the scope of nitrous oxide use amongst the teenage population. We reviewed previous published case studies along with statistics from the International Centre for Drug Policy. ${ }^{1}$

Results Pneumothorax and pneumomediastinum are recognised complications of illicit drug use. The underlying pathophysiology relates to barotrauma created by the inhalation technique. ${ }^{2}$

Inhalation of Nitrous Oxide has the added complication of pneumothorax expansion, due to its high blood-gas partition coefficient. Accordingly complications from the inhalation of nitrous oxide may be significantly worse than for users of other inhaled recreational drugs.

Recreational nitrous oxide use amongst the teenage population has risen exponentially over the past five years, with $7.6 \%$ of 16-24 year olds in England and Wales admitting to having tried nitrous oxide in 2013. ${ }^{3}$ Seventeen deaths in the UK were attributable to nitrous oxide between 2006-2012, ${ }^{1}$ a figure which we would expect to rise given current surge in usage.

Conclusion Nitrous oxide inhalation poses a significant risk to the teenage population. Pneumothorax should be considered in any teenager presenting after inhaling gaseous or volatile agents, and recreational drug use should be considered and addressed in teenagers presenting with a pneumothorax of unknown aetiology.

\section{REFERENCES}

1 John Corkery HC, Loi B, Goodair C, Schifano F. Drug Related Deaths in the UK: Jan-Dec 2012-2013

2 Phillipe Camus ECR. Drug-induced and latrogenic Respiratory Disease: CRC Press; 2010. 364p

3 Office H. Drug Misuse: Findings from the 2013/14 Crime Survey for England and Wales. www.gov.uk: Government, ONS; 2014 15/08/2014.

\section{G91(P) SALICYLATE LEVELS IN PAEDIATRIC INTENTIONAL OVERDOSE - ARE WE OVER INVESTIGATING?}

J Daniel, S Elliot. Paediatrics, Heart of England Foundation NHS Trust, Birmingham, UK

10.1136/archdischild-2015-308599.90 